\title{
Der Mehrwert der Sozialen Arbeit
}

\section{Eine Social-Return-on-Investment-Analyse am Beispiel eines Altenhilfeträgers}

\author{
WOLFGANG WASEL, KLAUS \\ GÜNTHÖR, ACHIM HOLLENBACH \\ Prof. Dr. Wolfgang Wasel hat eine \\ Professur für Gesundheitsmanage- \\ ment an der Hochschule Ravens \\ burg-Weingarten. \\ wolfgang.wasel@hs-weingarten.de
}

Klaus Günthör ist Prokurist der Altenhilfegesellschaften der Stiftung Liebenau in Meckenbeuren. klaus.guenthoer@st.anna-hilfe.de

Achim Hollenbach ist Doktorand der Pflegewissenschaft Universität UMIT/Hall in Tirol und Leiter der Abteilung Qualitätsmanagement der Altenhilfe der Stiftung Liebenau. achim.hollenbach@st.anna-hilfe.de

\author{
Die Methode des Social Return on Investment kann eine \\ Vorstellung davon vermitteln, welchen wirtschaftlichen \\ "Mehrwert" Soziale Arbeit haben kann. Eine Studie im \\ Auftrag der Stiftung Liebenau wandte diesen Ansatz \\ auf die drei Altenhilfegesellschaften der Organisation \\ an. Fazit: Die volkswirtschaftlichen "Gewinner" \\ sind Bund, Land, Gemeinden und Krankenkassen, \\ die "Verlierer" die Kreise und die Pflegekassen.
}

In diesem Artikel wollen wir versuchen, den Mehrwert eines Altenhilfeträgers darzustellen. Der Mehrwert soll auf der Basis des Social Return on Investment untersucht werden. Im Fokus steht eine Transferkostenanalyse der Finanzierungsträger (Bund, Land, Landkreis, Städte und Gemeinden sowie die Pflege- bzw. Krankenversicherungskassen; SROI 1 und 2). Die Ergebnisse zeigen, dass Krankenkasse, Bund, Länder und Gemeinden von dem Social Return on Investment erheblich profitieren. Dagegen sind die Kreise und die Pflegekassen die Verlierer. Es zeigt sich auch, dass der untersuchte Altenhilfeträger durch Beschäftigung, Investition und Konsum eine Wertschöpfung von ca. 164 Millionen Euro erzeugt und über 2.250 Arbeitsplätze schafft. Die Studie wurde im Auftrag der Stiftung Liebenau durchgeführt. Die Daten beziehen sich auf die drei Altenhilfegesellschaften in Deutschland der Stiftung Liebenau.

\section{Die Sozialwirtschaft braucht ein neues Maß}

Die letzten zwei Jahrzehnte der Sozialwirtschaft waren geprägt von der Einführung des betriebswirtschaftlichen Denkens und Handelns. Auslöser dieser Entwicklungen waren die finanziellen Situationen der sozialen Sicherungssysteme.
Die Träger der Sozialleistungen waren und sind kaum mehr in der Lage, ihre Aufgaben ohne Ausweitung ihrer Finanzierungsbasis und Einsparungen zu bewältigen. Verantwortlich für diesen Trend sind in erster Linie demografische Entwicklungen, konjunkturelle Schwierigkeiten und technisch-fachliche Fortschritte. Auswirkungen auf die Leistungserbringer sind mehr Wettbewerb, größeres Refinanzierungsrisiko, schlechtere Finanzierung der Leistung und geringere Investitionskostenförderung.

Zur Sicherstellung ihres Auftrages war die Sozialwirtschaft gezwungen, sich verstärkt mit betriebswirtschaftlichen Fragen zu beschäftigen (Wasel, 2011). Dies führte in allen Facetten von sozialwirtschaftlichen Unternehmen zu einem Boom wirtschaftlichen Denkens (Buestrich \& Wohlfahrt, 2008).

Oft negativ von Mitarbeitern und anderen Stakeholdern beäugt, sichert die Einführung der wirtschaftlichen Handlungsdimension den Sozialunternehmen das Überleben und ermöglicht nachhaltiges Arbeiten. Damit einher ging auch die Entwicklung von klassischen Kennzahlensystemen (Du Pont Schema etc.) zur Steuerung der Sozialunternehmen, die stark an den Systemen der freien Wirtschaft orientiert waren.

Mit einer gewissen Zeitverzögerung etablierten sich aber auch immer mehr 


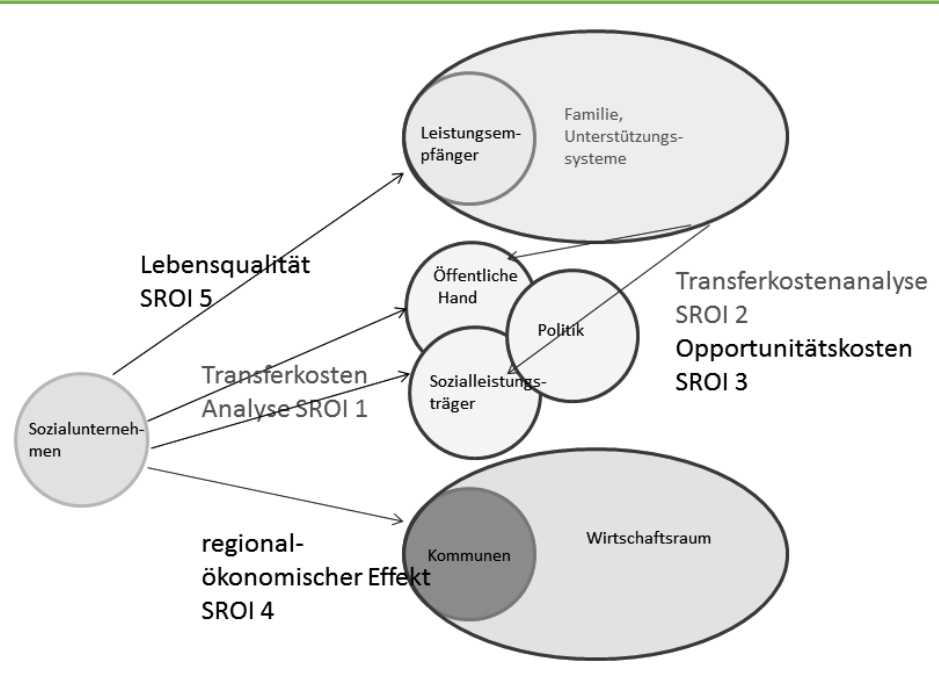

Abb. 1: Sozialer Mehrwert kann viele Gesichter haben.

Quelle: Schellberg, 2010

Kennzahlen, die versuchten, das »Proprium «, die fachliche Arbeit, abzubilden. Diese Kennzahlen finden sich in Qualitätsmanagementsystemen (EFQM; DIN ISO etc.), Aufbau von fachlichen Controllingsystemen, externen Kontrollverfahren (MDS Prüfrichtlinien; Prüfrichtlinien der Heimaufsicht etc.), Versuche, den Gemeinnutzen eines Unternehmens zu erfassen (Meynhardt \& Metelmann, 2009) oder aber sozio-ökonomische Analysen.

Daraus ergab sich für Unternehmen häufig ein fragmentarisches Controllingsystem. Was bislang also gegenüber klassischen (rein wirtschaftlichen) Kennzahlensystemen fehlt, ist eine eigenständige und durchgängige Ordnung des Zusammenspiels von sozialen und monetären Daten. Mit dem Ansatz des Social Return on Investment (SROI) wird diese Problematik überwunden.

\section{Der SROI-Ansatz}

Der Ansatz von Social Return on Investment geht ursprünglich auf Überlegungen des Robert Enterprise Funds Anfang der 1990er Jahre zurück (Aeron, 2004). Er hatte zum Ziel, den wirtschaftlichen und sozialen Mehrwert der Sozialen Arbeit zu erfassen. Im SROI Ansatz wird reflektiert, dass soziales Handeln nicht nur eine eineindeutige Wirkung (monokausal) hat, sondern in einer Wechselwirkung zwischen Sozial- und Gesundheitsunternehmen, Leistungsempfänger, Gesellschaft und Kostenträger steht.
Sozialunternehmen bemächtigen durch ihre Sozial- und Gesundheitsdienstleistung die Leistungsempfänger, am gesellschaftlichen Leben teilzuhaben. Die Gesellschaft profitiert davon materiell und immateriell. Anders formuliert: Menschen können am (Arbeits-)Leben (so weit möglich) wieder teilnehmen und Sozialunternehmen sorgen für mehr soziale Gerechtigkeit. Die Mitglieder einer Gesellschaft finanzieren durch ihre Sozialbeiträge die sozialen Sicherungssysteme. Die sozialen Sicherungssysteme wiederum finanzieren die Sozialunternehmen in weiten Teilen.

Wie von Schellberg (2010) beschrieben hat der Social Return on Investment viele Gesichter (vgl. Abb. 1). Der Mehrwert kann in fünf Aspekte gegliedert werden:

- Der Mehrwert für die Leistungsempfänger und ihr direktes Umfeld.
Dieser Wert wird im weiteren Sinne als sozial-klimatische Lebensqualität verstanden (SROI 5).

- Der Mehrwert, der für eine Region entstehen kann (SROI 4) und der Mehrwert, der für einzelne Finanzierungsträger ermittelt werden kann.

- Dieser wird in der Regel über die Opportunitätskosten (SROI 3) bzw. Transferkostenanalysen berechnet.

- In unserer Untersuchung interessieren wir uns für die Kosten-Nutzen Analyse aus der Sicht der öffentlichen Hand (SROI 1)

- und der Sozialleistungsträger (SROI 2).

Die Analyse, ob für die Finanzierungsträger ein Mehrwert entsteht, wurde durch eine Transferkostenanalyse der Finanzierungsträger (Bund, Land, Landkreis, Städte und Gemeinden sowie die Pflege- bzw. Krankenversicherungskassen) vorgenommen (SROI 1 und 2). In diesem Artikel soll nur der SROI 1 und 2 dargestellt werden. Die gesamte Untersuchung erfasste soziale und monetäre Aspekte (SROI 1,2,4 und 5; (Wasel, Hollenbach \& Günthör, Veröffentlichung in Vorbereitung)

\section{Transferkostenanalyse (SROI 1 und 2)}

Bei der Transferkostenanalyse sollen die Kosten aus der Perspektive der kommunalen Ebene, des Landes, des Bundes und der Träger der Sozialen Pflegeversicherung und der Krankenkassen in Relation zu ihren Einnahmen gestellt werden. Die Einnahmen werden einmal im Hinblick auf die Beschäftigten im Bereich der Altenhilfe dargestellt (volks-

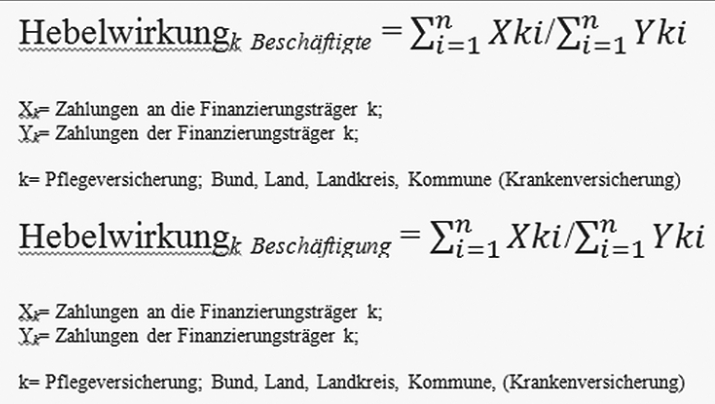

Abb. 2: Zur Ermittlung des volkswirtschaftlichen Nutzens (Modell I und II, Beschäftigte und Beschäftigung in der Altenhilfe) steht ein erprobtes Berechnungsmodell zur Verfügung. 


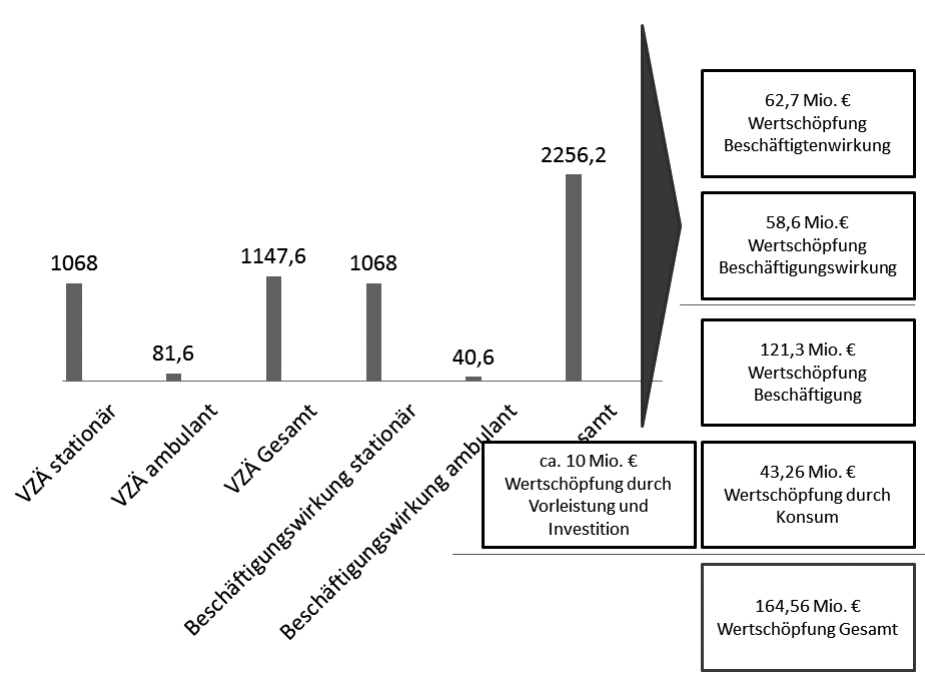

Abb. 3: Die Altenhilfe hat einen großen Wertschöpfungseffekt, der alleine im stationären und ambulanten Bereich gesamt über 2.250 vollzeitäquivalente Stellen schafft.

wirtschaftlicher Nutzen I) und in Bezug auf ihre Beschäftigungswirkung, also wie viele Menschen können aufgrund der Altenhilfeangebote ihrer Arbeit nachgehen (volkswirtschaftlicher Nutzen II). Die Kosten-Nutzen Relation wird anhand ihrer Hebelwirkungen dargestellt. Hebelwirkungen $>1$ stellen ein positive Kosten-Nutzen Relation dar (vgl. Abb. 2). Zur Berechnung der Zahlungsströme wurden folgenden Annahmen getroffen:

- Zahlungen der Länder (Investitionskostenförderung)

- Zahlungen der Kreise (Sozialhilfe und Förderung der Kreise im Jahresschnitt; bestimmt über die durchschnittliche, jährliche Abschreibung)

- Zahlungen der Gemeinden (Grundstücke in der Regel nicht mehr zugestiftet, sondern unentgeltlich gepachtet oder finanziert zu 0)

- Zahlungen der Mitarbeiter der Altenhilfe (Echtdaten)

- Einkommenssteuerzahlungen (ohne weitere individuelle Abgrenzungen) $* 42,5 \%$ (= Bundesanteil)

- Einkommenssteuerzahlungen (ohne weitere individuelle Abgrenzungen) *42,5\% (= Landesanteil)

- Einkommenssteuerzahlungen auf Landkreis-Ebene $(0,31$ (= durchschnittliche Kreisumlage im Jahr 2010) *Gemeindesteueranteil + 0,0343 (Anteil der vom Land an die LK von der Einkommenssteuer wieder zurückfließt)*(Einkommenssteuer Land)
- Einkommenssteuerzahlungen (ohne weitere individuelle Abgrenzungen) *15\% (Gemeindeanteil; Differenzierungen (Schlüsselbestimmungen) unterbleiben, da über alle Gemeinden der Effekt bei 15\% liegen muss).

- Zur Berechnung der Einkommenssteuer wurde pauschal über alle Mitarbeiter in der Altenhilfe der Stiftung Liebenau mit der Einkommenssteuerklasse I gerechnet.

- Zusätzliche Annahmen Berechnungsmodell des volkswirtschaftlichen Nutzens (Modell II; Beschäftigungswirkung)
- Pro Pflegenden (stationär) kann eine Person $(1,0)$ zusätzlich arbeiten gehen. Im ambulanten Bereich wurden 0,5 Personen angenommen, die durch das Angebot arbeiten gehen können. Als durchschnittlicher Lohn wurde die Angabe des Statistischen Landesamtes Baden Württemberg herangezogen. Es lag im Jahr 2010 bei 45.150 Euro (Arbeitnehmerbrutto). Da die Zuzahlungen der Grundsicherung im Alter nicht exakt bestimmt werden konnten, wurde näherungsweise auf ca. 9\% ausgewichen (vgl. Görtz, 2005). Ebenso wurde in beiden Modellen die Annahme getroffen, dass für private Pflegeversicherungen der Anteil bei ca. $10 \%$ liegt.

\section{Ergebnisse: der Mehrwert für die Finanzierungsträger (SROI 1 und 2)}

Die volkswirtschaftlichen Effekte wurden nach Finanzierungsträger, nach Beschäftigungs- und Beschäftigtenwirkung analysiert. Zunächst wird unabhängig vom SROI Modell der Wertschöpfungseffekt der gesamten Altenhilfe der Stiftung Liebenau dargestellt (vgl. Abb. 3).

Die Altenhilfe hat einen großen Wertschöpfungseffekt, der alleine im stationären und ambulanten Bereich gesamt über 2.250 vollzeitäquivalente Stellen schafft (1.147,6 Stellen direkte

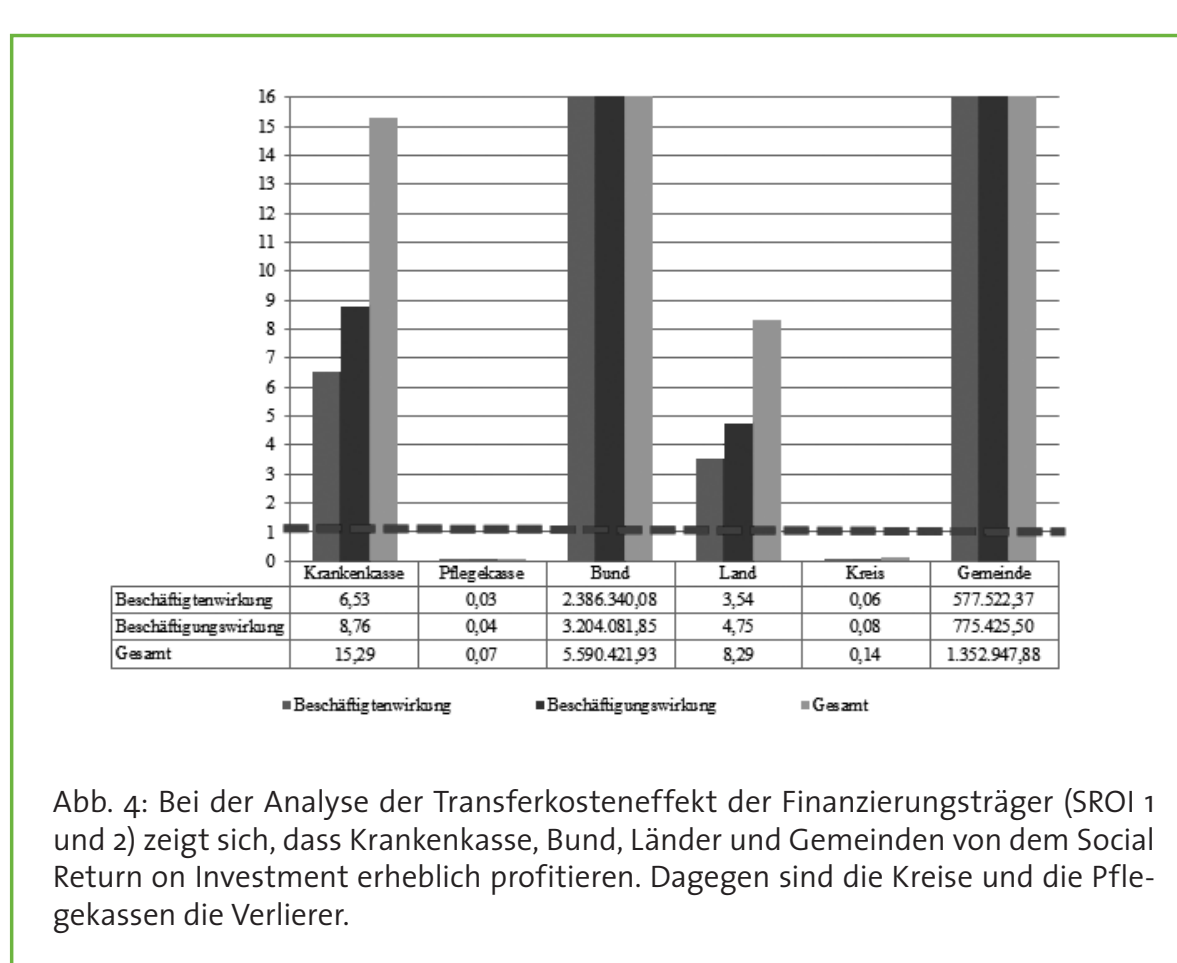


Beschäftigungswirkung und 1.108,6 Stellen indirekte Beschäftigungswirkung). Dabei wird eine Wertschöpfung von ca. 121 Millionen Euro erzeugt. Rechnet man noch zusätzliche Investitionskosten und Konsumeffekte hinzu (Berechnung erfolgte wie in Prognos, 2011) so ergibt sich eine Wertschöpfung von mehr als 160 Millionen Euro.

In den Transferkostenanalysen ergibt sich ein differenziertes Bild für die Finanzierungsträger. Es zeigt sich, dass Krankenkasse, Bund, Länder und Gemeinden von dem Social Return on Investment erheblich profitieren. Dagegen sind die Kreise und die Pflegekassen die Verlierer (vgl. Abb. 4).

An die Kreise fließen von jedem Euro 14 Cent zurück und von jedem Euro der Pflegekassen verbleiben für sie lediglich 7 Cent. Hauptprofiteure sind interessanterweise das Land, das von jedem investierten Euro 8,29 Cent zurückbekommt und die Krankenkassen, an die von jedem gezahlten Euro 15,29 Euro zurückfließen. Alle anderen Kostenträger profitieren deshalb so erheblich, weil sie keine oder kaum Zahlungen, aber erheblich von der Einkommenssteuer partizipieren.

Beide Effekte können aber auch dahingehend interpretiert werden, dass die zuständigen Hauptfinanziers ihrem Auftrag voll und ganz nachkommen. $\mathrm{Da}$ es sich um einen solidarischen Effekt handelt, ist es naheliegend, dass die "Verlierer« sich auch noch durch andere Personengruppen oder aber Zuwendungen, Steuern etc. finanzieren müssen. Gesamtbetrachtungen sind mit Vorsicht $\mathrm{zu}$ interpretieren, da es zwingend über alle Finanzierungsträger hinweg zu ausgeglichenen Haushalten kommen muss. Näherungsweise müssen daher bei großen Erhebungseinheiten die Hebelwirkungen über alle Bereiche der Altenhilfe zu 1 tendieren.

\section{Zusammenschau}

Der Ansatz des Social Return on Investment vermittelt ein Abbild des Mehrwerts der Altenhilfe: Dabei zeigt sich aus der Transferkostenanalyse ein differenziertes Bild bei den Finanzierungsträgern. Die volkswirtschaftlichen Gewinner sind Bund, Land, Gemeinden und Krankenkassen. Die Verlierer sind die Kreise und die Pflegekassen. Insgesamt ergibt sich ein Transferkostenef- fekt der nur knapp unter 1 liegt. Dies spiegelt die Zuständigkeiten des Solidarsystems wieder. Die Hauptfinanziers sind die Lastenträger, und es scheint ein funktionierendes Finanzausgleichssystem zu geben.

Der SROI Ansatz vermittelt damit eine vertiefte Analyse der Wirkungsebenen der Altenhilfe. Damit wird die Funktion der Altenhilfe in verschiedenen Facetten dargestellt. Es bietet daher auch die Grundlage für eine Sozialbilanz. Darüber hinaus besitzt es als Ausgangsbasis für verschiedene Denk- und Steuerungsmodelle sozialer Unternehmungen eine hohe Anschlussfähigkeit, als konzeptionelles Dach für Qualitätsmanagementsysteme (EFQM; DIN ISO; MDK etc.), für die Balanced Scorecard (Mitarbeiter, Produkt, Kunden, Finanzen etc.), für die strategische Steuerung nach sozio-ökonomischen Gesichtspunkten, für die strategische Steuerung nach Nachhaltigkeitsaspekten (Sustainability) oder für die strategische Steuerung aus Sicht der Corporate Social Responsibility.

Der SROI Ansatz sollte aber nicht dahingehend missbraucht werden, dass zukünftig nur noch Leistungen erbracht und finanziert werden, wenn sie eine positive Kosten-Nutzen Relation erzeugen. Die SROI Analyse vermittelt lediglich ein vertieftes Bild hinsichtlich des Mehrwertes sozialer Leistungen. Solidarisches Handeln ist vermutlich gerade dort notwendig, wo sich keine positiven Kosten-Nutzen Relationen ergeben. Außerdem sollte auch dieser Ansatz nicht hinweg darüber hinwegtäuschen, dass er unter einer Vielzahl an Annahmen berechnet wird und daher mit erheblichen Varianzen versehen sein muss.

\section{Literatur}

Aeron-Thomas, D. et al. (2004): Social Return on Investment. Valuing what matters. New economics foundation. London

Buestrich, M. \& Wohlfahrt, N. (2008). Die Ökonomisierung der Sozialen Arbeit. Aus Politik und Zeitgeschichte, 55,(12/13). S. 17-24.

Enste, D. H. (2009). Die Pflegedienstleistung - vom Kostenfaktor zum Jobmotor. Studie für den Bundesverband privater Anbieter sozialer Dienste e. V. Köln.

Görtz, J. (2005). Die Zukunft im ambulanten Pflegebereich. Vortrag beim Bundesverband privater Anbieter sozialer Dienste e. V. Köln Halfar, B. (2010). Social Return on Investment.
Ein Verfahren zur Berechnung der gesellschaftlichen Wertschöpfung der sozialen Unternehmen und Einrichtungen. Vortrag beim Bundesverband der Behindertenhilfe e. V. Berlin.

Meynhardt, T., \& Metelmann, J. (2009). Pushing the Envelope: Creating Public Value in the Labor Market: An Empirical Study on the Role of Middle Managers. International Journal of Public Administration, 32(3/4): S. 274-312.

Prognos AG (2011). Der volkswirtschaftliche Nutzen der Reha-Kliniken in Baden-Württemberg. Basel.

Schellberg, K. (2010). Der Mehrwert des Sozialen. Möglichkeiten zur Bestimmung der wirtschaftlichen Wirkung von Sozialunternehmen. Vortrag auf der INAS-Tagung. Linz.

Wasel, W. (2011). Rebound Effects in der Sozialwirtschaft. Vom Sozialen zur Wirtschaft und wieder zurück. Gesundheits- und Sozialpolitik, S. 25-31.

Wasel, W., Hollenbach, A. \& Günthör, K. (in Vorbereitung). Ambulant vor stationär - eine sozioökonomische Analyse.

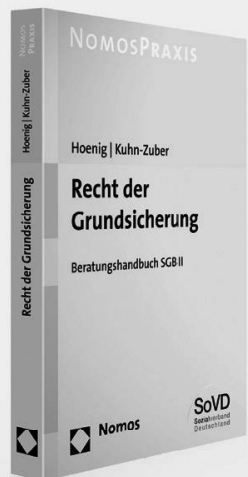

Recht der Grundsicherung Beratungshandbuch SGB II Von Ragnar Hoenig und Prof. Dr. Gabriele Kuhn-Zuber 2012, 365 S., brosch., 49,-€ ISBN 978-3-8329-6770-3

"sollte auf den Schreibtischen vieler Sozialberaterinnen und Sozialberater, in Beratungszentren und Sozialrechtskanzleien nicht mehr fehlen."

SoVD-Zeitung $7+8 / 12$

www.nomos-shop.de/13868

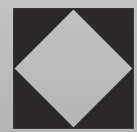

Nomos 